\title{
Application of Monoclonal Antibodies to Measure Metabolism of an Anti-Trypanosomal Compound In Vitro and In Vivo
}

\author{
Rachel Beaulieu Goldsmith, ${ }^{1}$ Danny R. Gray, ${ }^{2}$ Zhixia Yan, ${ }^{3}$ Claudia N. Generaux, ${ }^{3}$ \\ Richard R. Tidwell, ${ }^{1 *}$ and Howard M. Reisner ${ }^{1}$ \\ ${ }^{1}$ Department of Pathology and Laboratory Medicine, School of Medicine, University of North Carolina, \\ Chapel Hill, North Carolina \\ ${ }^{2}$ Center for Delivery of Molecules and Cells, Department of Biomedical Engineering, Case Western \\ Reserve, Cleveland, Ohio \\ ${ }^{3}$ Eshelman School of Pharmacy, University of North Carolina, Chapel Hill, North Carolina
}

\begin{abstract}
Human African trypanosomiasis (HAT), also called African sleeping sickness, is a neglected tropical parasitic disease indigenous to sub-Saharan Africa. Diamidine compounds, including pentamidine and CPD-0801, are potent anti-trypanosomal molecules. The latter is a potential drug in the development at the UNC based Consortium for Parasitic Drug Development. An orally bioavailable prodrug of CPD-0801, DB868, is metabolized primarily in the liver to the active form. A monoclonal antibody developed against a pentamidine derivative has shown significant reactivity with CPD-0801 (EC $5065.1 \mathrm{nM})$, but not with the prodrug $\left(E_{50}>18,000 \mathrm{nM}\right)$. An inhibitory enzyme-linked immunosorbent assay

(IELISA) has been used to quantitatively monitor prodrug metabolism by detecting the production of the active compound over time in a sandwich culture rat hepatocyte system and in rats. These results were compared with the results of the standard LC/MS/MS assay. Spearman coefficients of 0.96 and 0.933 (in vitro and in vivo, respectively) indicate a high correlation between these two measurement methods. This novel IELISA provides a facile, inexpensive, and accurate method for drug detection that may aide in elucidating the mechanisms of action and toxicity of existing and future diamidine compounds. J. Clin. Lab. Anal. 24:187-194, 2010. (C) 2010 Wiley-Liss, Inc.
\end{abstract}

Key words: immunoassay; ELISA; pentamidine; trypanosomiasis; pafuramidine; CPD

Human African Trypanosomiasis (HAT; also known as sleeping sickness) is a fatal parasitic disease indigenous to sub-Saharan Africa. HAT is largely found in remote rural areas where it is caused by unicellular flagellated protozoa of the Trypanosoma brucei sub species. After the introduction of the organism to the host blood stream by the tsetse fly, HAT progresses through two increasingly severe stages. In the first stage, parasites are localized to the circulation resulting in flulike symptoms, which are often ignored (1). If untreated, the disease will progress to the second stage characterized by central nervous system infection leading to coma and death $(1,2)$.

Current treatments for HAT are problematic because they must be delivered parenterally, are often effective for only a single stage of disease, and have significant toxic side effects (3). The most common treatment for the first stage of disease is pentamidine (Fig. 1), a diamidine compound delivered intravenously or intramuscularly (3) that can cause dysglycemia and hepatoxicity. Melarsoprol, an arsenical compound, is the most common treatment for second-stage HAT. It must be delivered intravenously in a hospital setting because up to $10 \%$ of treated patients experience post-treatment reactive encephalopathy, which is fatal to half of the affected patients $(3,4)$.

Because no treatment for HAT is satisfactory, new compounds are in development. A series of diamidine

Grant sponsor: Department of Pathology Scholarly Activities Trust Fund.

*Correspondence to: Richard R. Tidwell, CB\# 7525 BBB805, University of North Carolina at Chapel Hill, Chapel Hill, NC 27599-7525. E-mail: tidwell@med.unc.edu

Received 7 December 2009; Accepted 19 March 2010

DOI $10.1002 /$ jcla. 20380

Published online in Wiley InterScience (www.interscience.wiley.com). 
<smiles>N=C(N)c1ccc(OCCCCCOc2ccc(C(N)=O)cc2)cc1</smiles><smiles>N=C(N)c1ccc(-c2ccc(-c3ccc(C(=N)N)cc3)o2)cc1</smiles><smiles>N=C(N)c1ccc2c(c1)[nH]c1cc(C(=N)N)ccc12</smiles>

Fig. 1. Diamidine compounds used for screening monoclonal antibodies.

compounds have shown mixed results. Pafuramidine, an orally bioavailable prodrug, metabolizes to the active parent, furamidine (Fig. 1), which proved effective in the first stage of disease. Unfortunately, Phase III clinical trials of pafuramidine have been halted due to an extended Phase I study, which revealed delayed nephrotoxicity $(5,6)$. Another diamidine analog, CPD0801, and its prodrug DB868, are currently in development to treat the second stage of HAT (7).

A commonly used method in small molecule drug development is high-performance liquid chromatography/mass spectroscopy (LC/MS/MS). Although effective in the research laboratory, LC/MS/MS cannot easily be implemented in the clinics of Africa, where ultimately the bioefficacy studies will be assessed. Protein-based methodologies, specifically enzyme-linked immunosorbent assays (ELISA), could be utilized to study small molecules. This technique is less expensive and more facile than traditional small molecule assays.

Polyclonal antibodies were originally developed against a pentamidine analog (1-[4-amidinophenoxy]-5-[4-aminophenoxy]pentane, ADAMP) for use in an inhibitory ELISA (IELISA) (8). Subsequently, monoclonal antibodies (Mabs) were produced against the same antigen to provide a more uniform source of high-affinity reagents for use in the IELISA. The availability of Mabs directed against diamidines has led to the development of a simple assay for diamidines of potential clinical interest. As demonstrated in this article, Mab-based immunoassays can be used to detect CPD-0801 and distinguish it from its prodrug both in vitro and in vivo. This method could be further applied to studying the metabolism of new antiHAT agents and localizing the active metabolites in tissue.

\section{MATERIALS AND METHODS}

\section{Production of Mabs}

Immunization

Two-month-old female BALB/c mice (Charles River, Raleigh, NC) were immunized with pentamidine-ovalbumin conjugate $(0.2 \mathrm{mg} / \mathrm{ml}$ protein, hapten-carrier ratio of 23) prepared as described by Reisner et al. (8). Antigen was emulsified into TitermaxTM adjuvant (CytRx, Norcross, GA) according to the product instructions. Primary immunization was performed by subcutaneous injection into the upper groin region with adjuvant solution containing $100 \mu \mathrm{g}$ of antigen. Two further immunizations were performed at 2-week intervals by intraperitoneal (i.p.) injection of $100 \mu \mathrm{g}$ antigen diluted in phosphate-buffered saline (PBS). One week after the third immunization, plasma was collected from anesthetized animals and screened using ELISA methodology as previously described (8) with modifications described below. Mice demonstrated titers of 1:800 or greater (defined as last dilution demonstrating an optical density of at least 1.5). A final i.p. boost with $20 \mu \mathrm{g}$ of antigen was performed 5 days before fusion. All animal protocols were approved by the Institutional Animal Care and Use Committee (IACUC) of the School of Veterinary Medicine at North Carolina State University where fusions were performed. Animal experiments were approved by the IACUC of the University of North Carolina at Chapel Hill.

\section{Hybridoma production}

Myeloma cell line P3-X63-Ag8.653 (American Type Culture Collection, Rockville, MD) was used as a fusion partner. Standard fusion protocols using HAT selection were followed (9) with the following additions: Balb/c peritoneal feeder cell layers were used during initial expansion of selected clones from 96 well plates and during three rounds of cloning by limiting dilution. During these stages, media were also supplemented by the addition of low-serum supernatant from P388D cells $(10,11)$. Twelve days after fusion, macroscopic growth was present in $86 \%$ of wells seeded with $5 \times 10^{4}$ nucleated spleen cells per well. Initial screening of 800 wells detected significant reactivity $(\mathrm{OD}>0.30)$ in $12 \%$ using a screening ELISA against a pentamidine derivative, 31 of these were strong $(\mathrm{OD}>1.00)$.

\section{Immunoassay Design}

Detection of immunoreactivity (ELISA for pentamidine)

An ELISA assay for the detection of Mab reactivity toward pentamidine and related molecules was estab- 
lished based on the previous work (8) using ReactiBindTM maleic anhydride activated polystyrene 96-well "strip plates" (Pierce, Rockford, IL) sensitized with ADAMP (synthesized at UNC Chapel Hill) diluted to $0.01 \mu \mathrm{g} / \mathrm{ml}$ in PBS pH 7.4 (Sigma, St. Louis, MO) (as described in detail in Reisner et al. (8)). One hundred microliters of appropriate dilutions of animal sera, Mab containing supernatant or purified immunoglobulin preparations were made in PBSB (PBS with 2\% Blotto) and incubated in sensitized and blocked wells for $2 \mathrm{hr}$ at $37^{\circ} \mathrm{C}$ before washing and development with goat antimouse horseradish peroxidase labeled antibody (Jackson ImmunoResearch Laboratories Inc., West Grove, PA) diluted 1:20,000 in PBSB. The color change was monitored at $405 \mathrm{~nm}$ with a SPECTRAmax 340PC plate reader (Molecular Devices, Sunnyvale, CA). The natural fluorescence of the compounds does not interfere with this color change reaction.

\section{Inhibitory ELISA for the detection of pentamidine-like compounds}

The IELISA was carried out as in Reisner et al. (8) with the following modifications. Fifty microliters of analyte ("inhibitor") was mixed with $50 \mu \mathrm{l}$ of an appropriate dilution of Mab (generally 1:100 dilution of monoclonal anti-pentamidine antibody 1.85.72) in sensitized blocked wells and incubated for $2 \mathrm{hr}$ at room temperature (RT) followed by washing and addition of secondary antibody to the wells at RT with TrisBuffered Saline (TBS). Blank mouse plasma was included on each plate as a control for secondary antibody binding, and a vehicle "zero-dose" control for maximum binding was also included. Pentamidine-like compounds were assayed for immunoreactivity using dilutions from approximately $4 \mathrm{nM}$ to $16 \mu \mathrm{M}$ in DMSO. Two compounds were tested on each plate, one of which had been previously tested and therefore served as a control. The assay was replicated four times per compound on each plate for two plates; then repeated with a series of higher concentrations to more fully cover the relevant range of concentrations. The data were manually normalized to the "zero-dose" DMSO control. Inhibition curves were plotted and the $\mathrm{EC}_{50}$ calculated using a sigmoidal variable slope dose-response curve (Prizm version 4 GraphPad Software, Inc., San Diego, CA).

\section{Metabolite Detection}

Treatment of sandwich-cultured rat hepatocytes with DB868

Hepatocytes were isolated from male Wistar rats, and seeded onto BioCoatTM collagen plates as reported previously (12). Approximately $24 \mathrm{hr}$ after seeding, cells were overlaid with BD Matrigel ${ }^{\mathrm{TM}}$ (BD, Franklin Lakes, NJ). Thereafter, culture medium was changed for every $24 \mathrm{hr}$. On day 4, sandwich-cultured rat hepatocytes (SCRH) were incubated with DB868 $(10 \mu \mathrm{M})$ in culture medium. Medium samples were obtained at 0.08, 0.17, $0.33,0.5,1,2,4,8,16$, and $24 \mathrm{hr}$. All samples were stored at $-20^{\circ} \mathrm{C}$ until LC/MS/MS analysis. Animal experiments were approved by the IACUC of the University of North Carolina at Chapel Hill.

\section{Treatment of rats with DB868}

Male Sprague-Dawley rats were administered with DB868 $(25 \mu \mathrm{mol} / \mathrm{kg})$ formulated as a suspension of $0.1 \%$ Tween 80:ethanol $(80: 20 \mathrm{v} / \mathrm{v})$ by oral gavage. Blood $(0.15 \mathrm{~mL})$ was collected from a tail vein at $0.25,0.5,0.75$, $1,2,4,8,12,24$, and $48 \mathrm{hr}$ after DB868 administration. Plasma was stored at $-20^{\circ} \mathrm{C}$ until analysis by LC/MS/ MS. Animal experiments were approved by the IACUC of the University of North Carolina at Chapel Hill.

\section{Analysis of in vitro and in vivo samples by using LC/ $\mathrm{MS} / \mathrm{MS}$}

Analysis was modified from Midgley et al. and Trendler et al. $(13,14)$. All the data were acquired by using the ADME Mass Spectroscopy Center at UNC Chapel Hill (protocol to be published).

\section{IELISA analysis of in vitro and in vivo samples}

The IELISA was carried out for the detection of pentamidine-like compounds as above with the following modifications. Fifty microliters of medium or a 5 -fold dilution (in TBS $+2 \%$ blotto) of rat plasma was added to $50 \mu \mathrm{l}$ of a 1:100 dilution of Mab 1.85.72 in a sensitized blocked well and incubated for $2 \mathrm{hr}$ at RT followed by washing and addition of secondary antibody (as described above). The data were manually normalized to the vehicle "zero-dose" control. A nonparametric correlation test was performed between the LC/MS/MS data and the ELISA data (Prizm version 4 GraphPad).

\section{RESULTS}

\section{Production and Selection of Mabs}

Previous work from this laboratory established the technology for the production of murine polyclonal antibodies reactive with pentamidine and related compounds (8). Such antibodies show specificity for the aromatic amidine group of the immunizing hapten and broad cross-reactivity with other dicationic agents having related structures (Fig. 1) as detected using the 
IELISA. Based on this work, Mabs were generated to the pentamidine-ovalbumin conjugate to be used in developing a Mab-based IELISA. Candidate Mab clones were screened not only for reactivity with the parent compound (pentamidine) but also with 2,5-bis-[4amidinophenyl]furan (furamidine), and 2,7-diamidinocarbazole (02DAP092) (15) (see Fig. 1 for structures). CPD-0801 was not available in sufficient quantity for use at the time of screening. Supernatants from 12 of the 96 wells (chosen on the basis of titer) were evaluated for reactivity against furamidine and 02DAP092 in an IELISA format, and three wells were chosen for subcloning through two more selection cycles to monoclonality. The three resultant Mabs (clones 1.85.72, 1.93.65 and 4.57.72) all demonstrated some degree of reactivity with the target compounds and were of the $\mathrm{IgG}_{1}$ isotype. Individual cultures secreted between 100 and $160 \mu \mathrm{g}$ of immunoglobulin per milliliter of media. One Mab, 1.85.72, was chosen for detailed study as it showed the greatest sensitivity for the detection of pentamidine, and also demonstrated the greatest degree of cross-reactivity between the three diamidines (Fig. 2).

\section{Sensitivity and Specificity of Mabs}

Assay sensitivity and specificity were evaluated using the previously described IELISA format in which ADAMP was covalently coupled to microtiter plates. In this assay the ability of analyte to compete with the "sensitized" well for Mab 1.85.72 binding was measured as an indication of antigenic activity (Fig. 2). EC 50 values were determined for assays of the three target compounds using either assay buffer or a $1 / 6$ dilution of human serum in buffer as the sample matrix. As expected, the assay was most sensitive in detecting pentamidine $\left(\mathrm{EC}_{50}\right.$ in the $0.2-0.6 \mathrm{ng} / \mathrm{ml}$ range), less sensitive in detecting 02DAP092 $\left(\mathrm{EC}_{50}\right.$ of $\left.1.0-3.0 \mathrm{ng} / \mathrm{ml}\right)$, and least sensitive for furamidine $\left(\mathrm{EC}_{50}\right.$ about $\left.15.0 \mathrm{ng} / \mathrm{ml}\right)$. The assays for pentamidine and 02DAP092 were slightly more sensitive in the presence of diluted serum (details in Table 1). The assay sensitivity for the three dicationic agents was sufficient for potential use in a clinical setting. Mabs 1.93.65 and 4.57.72 (not studied in detail) had $\mathrm{EC}_{50}$ values for pentamidine in assay buffer of about 3.0 and $15.0 \mathrm{ng} / \mathrm{ml}$, respectively. The use of monoclonal anti-pentamidine antibodies significantly reduced the assay background compared with previous assays that used the polyclonal antibodies.

Mab 1.85.72 was evaluated for reactivity with bisamidoxime and methylamidoxime prodrug derivatives of furamidine (DB290 and pafuramidine, respectively). No reactivity was detected with either prodrug when tested at $100 \mathrm{ng} / \mathrm{ml}$. The prodrugs have less than $2 \%$ of the immuno reactivity of the parent compound (data not
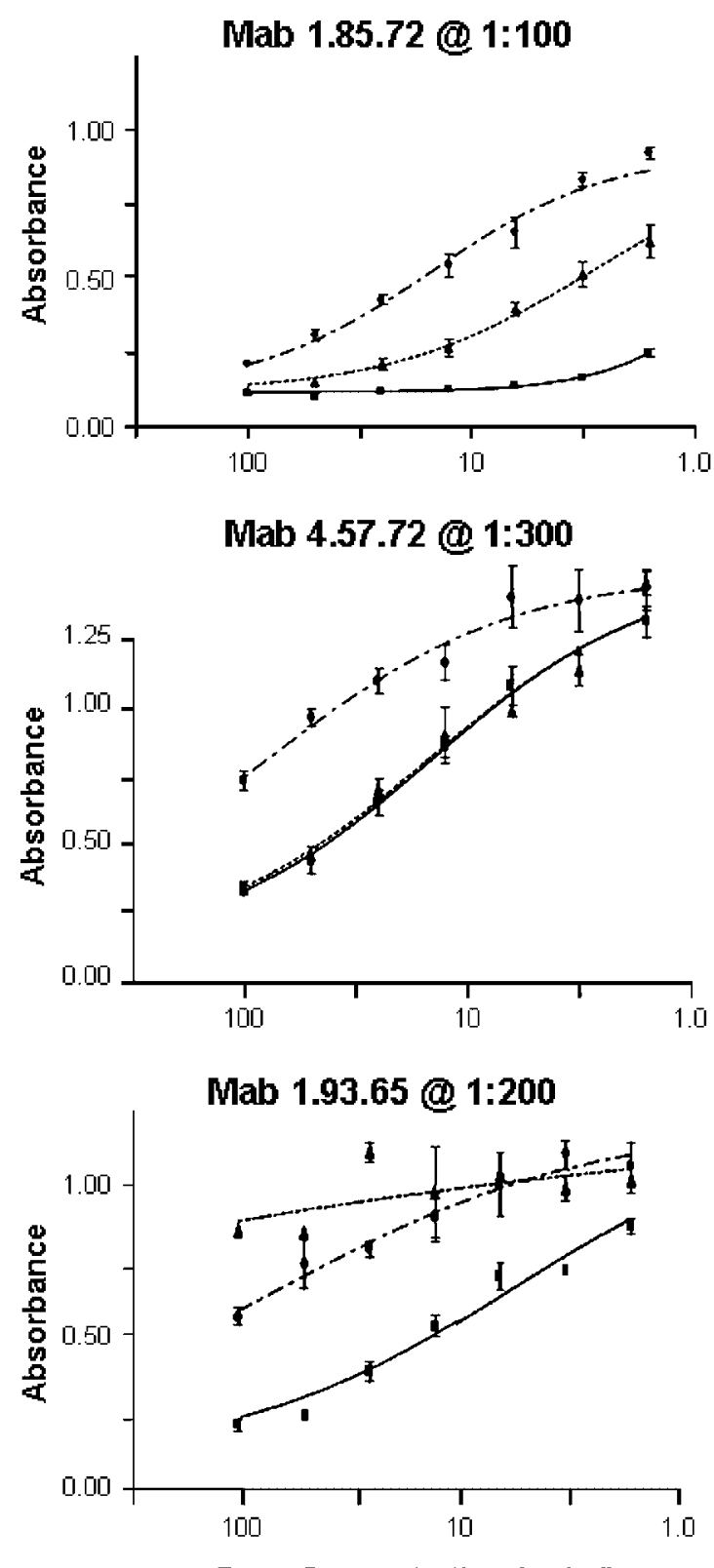

Drug Concentration (ng/ml)

Fig. 2. Cross-reactivity of different antibodies to the three screening compounds. Immunoassay for diamidine reactivity using pentamidine ( $\boldsymbol{\square})$, 02DAP092 ( $\mathbf{\Delta})$ and furamidine $(\bullet)$ standards diluted in PBS/blotto. Vertical bars represent standard deviation. Lines represent the best fit using non-linear regression as described in the Materials and Methods. The parameter representing maximal binding was estimated and fixed using " 0 -dose" controls.

shown) suggesting that the Mab can be used to monitor prodrug conversion.

\section{Detection of DB868 and Known Metabolites}

The IELISA was used to evaluate the utility of the Mab-based assays in detecting the bioconversion of the newly developed prodrug (DB868) to its active 
metabolite (CPD-0801, metabolic pathway shown in Fig. 3). The $\mathrm{EC}_{50}$ s were used as a basis of comparison of prodrug, intermediates, and active diamidine (Table 2). DB868, M1, and M2 have no significant immunoreactivity. Both M3 and CPD-0801 demonstrate significant immunoreactivity in the assay. The $\mathrm{EC}_{50}$ for CPD-0801 is approximately twice that of M3 but still approximately 16 times less then pentamidine. M4 could not be tested, as it is unstable.

TABLE 1. IELISA Sensitivity

\begin{tabular}{llccc}
\hline Compound & Matrix & $\mathrm{ED}_{50}$ & $95 \%$ CI & Sensitivity \\
\hline Pentamidine & Serum & 0.18 & $0.15-0.21$ & $>0.1$ \\
& Buffer & 0.55 & $0.37-0.82$ & ND \\
02 DAP092 & Serum & 1.3 & $1.1-1.5$ & $>0.5$ \\
& Buffer & 2.9 & $2.4-3.5$ & ND \\
Furamidine & Serum & 14.5 & $11.8-17.8$ & $>2.0$ \\
& Buffer & 15.0 & $12.9-17.5$ & ND \\
\hline
\end{tabular}

Immunoassay for comparing pentamidine, 02DAP092, and Furamidine using standards diluted in PBS/blotto or a $1 / 6$ dilution of human serum in $\mathrm{PBS} /$ blotto. $\mathrm{EC}_{50}$ calculations were made as described in the Materials and Methods. The data are derived using a four-parameter fit. $\mathrm{EC}_{50}$ is the concentration for "half" inhibition.

\section{Monitoring Metabolism of DB868 In Vitro}

After successfully differentiating between DB868 and the active diamidine, CPD-0801, the IELISA was utilized to detect DB868 metabolism in vitro. SCRH metabolized DB868 over time to CPD-0801. Metabolism was measured using the standard LC/MS/MS quantification of M3 and CPD-0801, the metabolites known to bind to Mab 1.85.72 (Fig. 4B). As expected, M3 initially increases rapidly, and CPD-0801 gradually increases over the 24-hr period. A similar trend is observed in the data acquired by IELISA (Fig. 4A). The non-parametric correlation yields the highest Spearman coefficient, 0.96, when the LC/MS/MS data for both M3 and CPD-0801 are combined in the comparison (Table 3).

\section{Monitoring Metabolism of DB868 In Vivo}

To evaluate the use of the IELISA for monitoring in vivo bioconversion of prodrug, Sprague-Dawley rats were orally treated with DB868. Metabolism was measured using the standard method, LC/MS/MS quantification of CPD-0801 (Fig. 4D), the only metabolite available for quantification at that time, in plasma

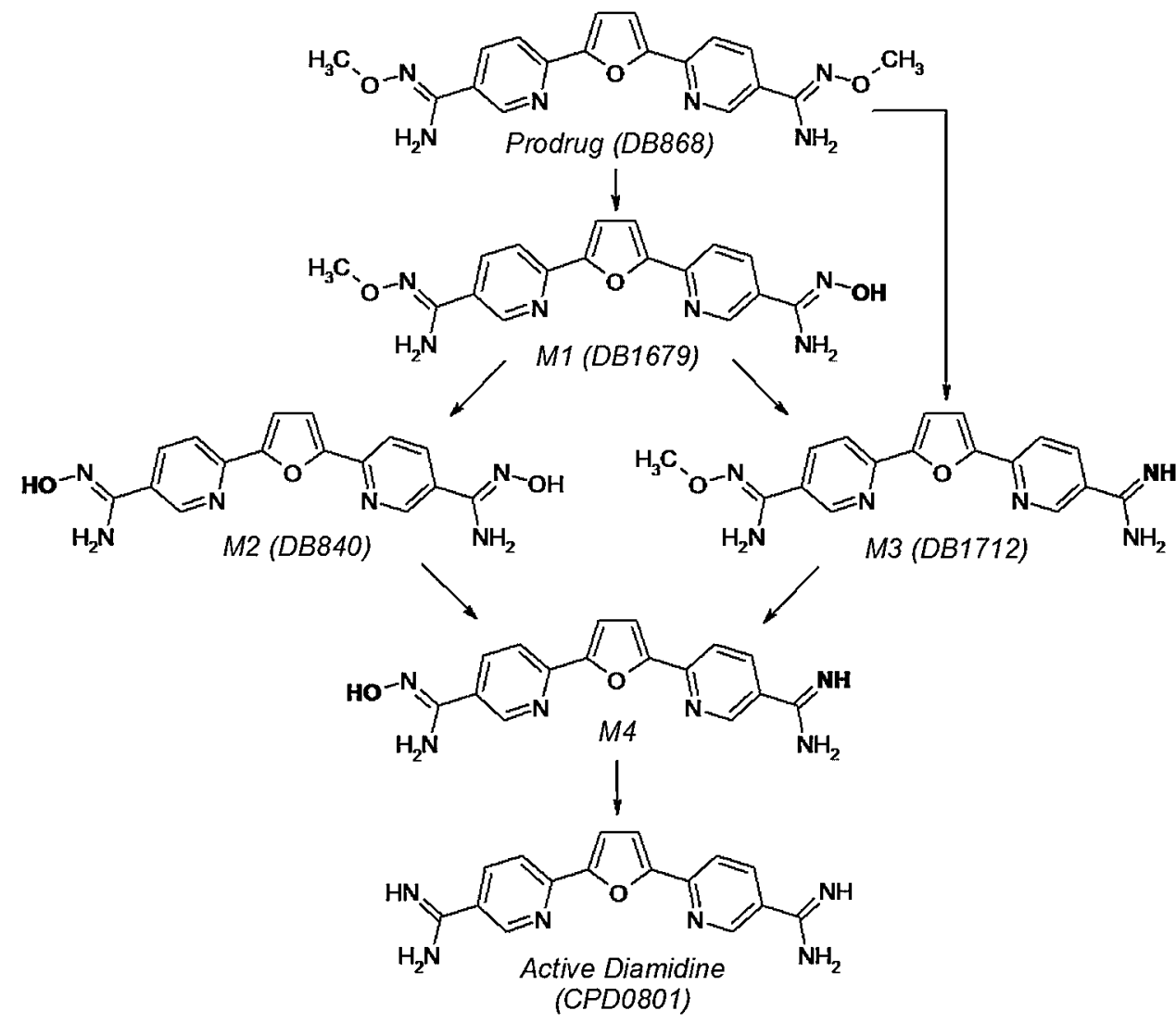

Fig. 3. Scheme depicting the metabolism of DB868 to CPD-0801. Adapted from (16). 
samples. CPD-0801 rapidly increases in the first $2 \mathrm{hrs}$ then decreases through the remainder of the 24-hr period. A similar, but not identical, trend is observed in the data acquired by IELISA (Fig. 4C). The presence of detectable compounds rapidly increases in the first $2 \mathrm{hr}$; however, it does not return to the initial value perhaps because the antibody detects M3, active diamidine, and

TABLE 2. EC $_{50}$ of Mab 1.85.72 Binding to DB868 and Metabolites

\begin{tabular}{lccl}
\hline Compound & $\mathrm{EC}_{50}(\mathrm{nM})$ & $\mathrm{SEM}^{\mathrm{a}}$ of $\mathrm{EC}_{50}$ & Binding? \\
\hline DB868 & $>18,000$ & - & Negligible \\
M1 & $>16,000$ & - & Negligible \\
M2 & $>16,000$ & - & Negligible \\
M3 & 123.7 & 0.3 & Yes \\
M4 & & Unstable & \\
CPD-0801 & 65.1 & 0.3 & Yes \\
Furamidine & 17.6 & 0.02 & Yes \\
Pentamidine & 4.07 & 0.01 & Yes \\
\hline
\end{tabular}

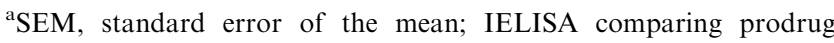
(DB868), M1, M2, M3, CPD-0801, furamidine, and pentamidine standards diluted in TBS/blotto. Attempts to synthesize M4 failed to result in a stable compound for quantification purposes.

possibly M4. The non-parametric correlation yields a Spearman coefficient of 0.93 .

\section{DISCUSSION}

Pentamidine has long been used as an anti-trypanosomal compound. However, its use is limited as it must be delivered parenterally, has potentially serious side effects, and can only treat the first stage of HAT. For these reasons, several other diamidine compounds are in development for the treatment of HAT, most notably CPD-0801 for the treatment of second-stage disease. To avoid parenteral dosing, an orally bioavailable prodrug (DB868) was developed, which is bioconverted in the liver to the active agent. Therefore, a method to specifically measure the quantity and tissue disposition of active metabolites in the presence of prodrug is of importance and may be accomplished using the immunoassay for pentamidine-like compounds described in this study. Although immunoassays would not be expected to have the exacting specificity and sensitivity of well-described LC/MS/MS-based assays (13), they have the potential advantage of simplicity and low cost.
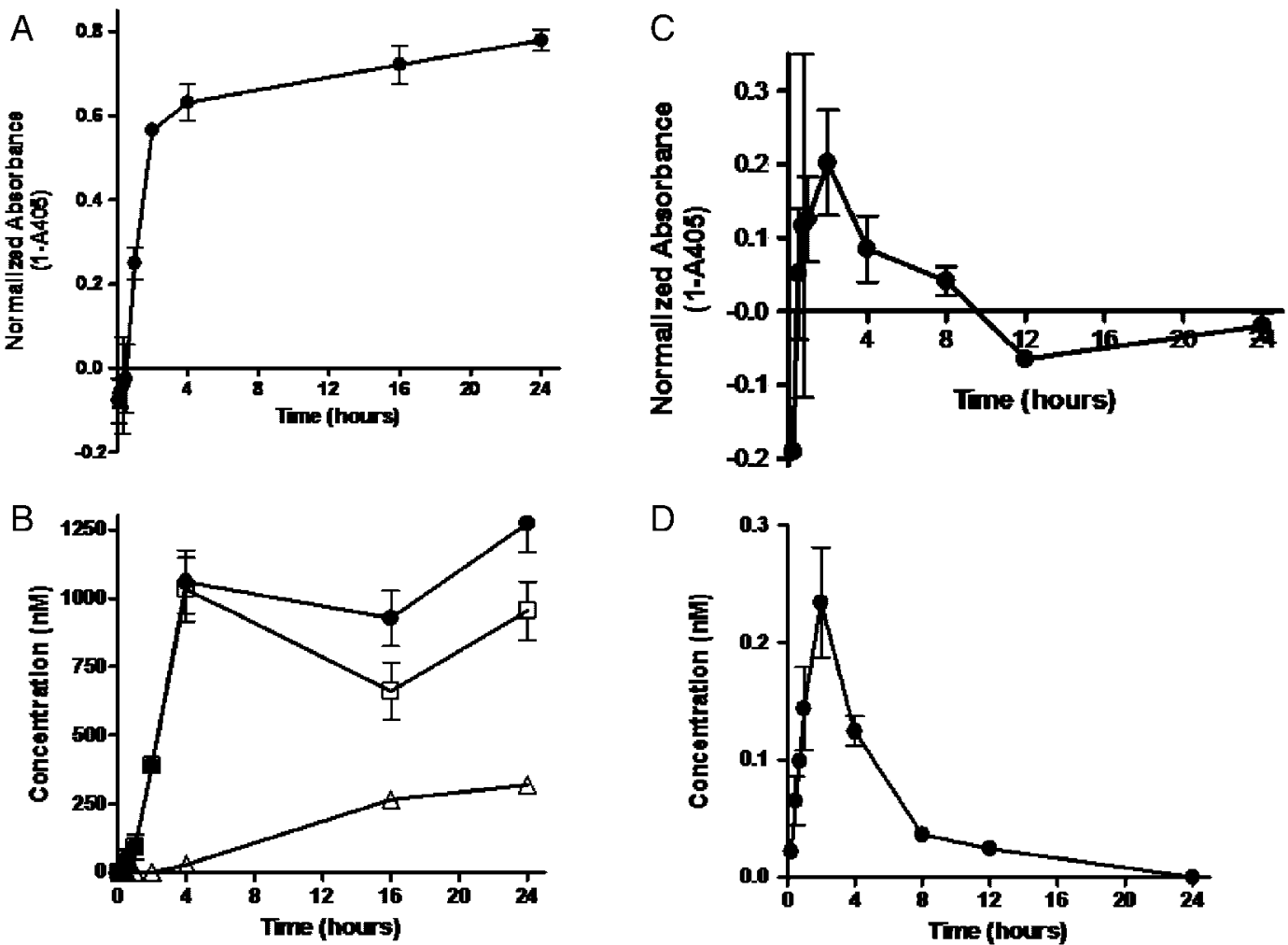

Fig. 4. LC/MS/MS and IELISA detection of DB868 metabolites in vitro and in vivo. (A) IELISA analysis of media from SCRH treated with DB868. Vertical bars show standard deviation. (B) LC/MS/MS quantification of M3 ( $\Delta$ ) and CPD-0801 ( $\square$ ) in media from SCRH. M3 added to CPD-0801 is also shown $(\bullet)$. Vertical bars show standard deviation. (C) IELISA analysis of plasma from rats treated with DB868. Vertical bars show standard deviation. (D) LC/MS/MS quantifiation of CPD-0801 in rats treated with DB868. Vertical bars show standard error. 
TABLE 3. Correlations Between IELISA and LC/MS/MS

\begin{tabular}{lll}
\hline & \multicolumn{1}{c}{ Comparison } & Spearman coefficient \\
\hline \multirow{2}{*}{ In vitro } & M3 vs. IELISA & $0.92^{*}$ \\
& CPD-0801 vs. IELISA & $0.88^{*}$ \\
\multirow{2}{*}{ In vivo } & M3 + CPD-0801 vs. IELISA & $0.96^{*}$ \\
\hline
\end{tabular}

Compound names indicate the molecule quantified by LC/MS/MS. ${ }^{*} P \leq 0.0031 ;{ }^{* *} P=0.007$.

The assay sensitivity for the three Mabs studied was sufficient for potential use in a clinical setting. Mabs 1.93.65 and 4.57.72 (not studied in detail) had $\mathrm{EC}_{50}$ values for pentamidine in assay buffer of about 3.0 and $15.0 \mathrm{ng} / \mathrm{ml}$, respectively. The use of monoclonal antipentamidine antibodies significantly reduced the assay background compared with previous assays that used the polyclonal antibodies. Mab 1.85.72 was selected for use in all subsequent experiments because it most effectively detected furamidine $\left(\mathrm{EC}_{50} 15 \mathrm{ng} / \mathrm{ml}\right)$.

We have used the Mab-based assay in ongoing studies of the newly developed prodrug/drug pair DB868 and CPD-0801 (7). As shown in Table 2, only active diamidine, CPD-0801, and M3 had significant immunoreactivity in the Mab-based assay. This suggests that the amidine moiety is essential for antibody recognition, and if M4 were tested, the antibody would most likely bind to it with a similar affinity to that of M3. However, there remains a 16-fold difference in affinity between CPD-0801 and pentamidine, both diamidines; therefore, other compounds would need to be screened to ascertain the importance of the central portion of the molecule to antibody binding. It is advantageous that the monoclonal antibody recognizes only those DB868-derived compounds, which have activity against trypanosomes (7).

The IELISA can be used to monitor the prodrug metabolism to active compound(s). SCRH were treated with DB868. Medium was monitored for M3 and CPD0801 by $\mathrm{LC} / \mathrm{MS} / \mathrm{MS}$ and the results were compared with the medium analysis by the IELISA (Fig. 4). The two techniques had the strongest correlation, as defined by a Spearman coefficient of 0.96 , when the LC/MS/MS data for M3 and CPD-0801 were added together. This is not surprising as it has been established that the antibody binds both to M3 and active diamidine.

Metabolism can be measured in an in vitro system, but for the assay to be clinically relevant, the antibody must detect active diamidine compound in a plasma matrix. Rats were orally treated with DB868, and the active diamidine was measured in plasma over time by LC/MS/MS. The standard analysis was compared with the IELISA, resulting in a Spearman coefficient of 0.93 . The correlation is satisfactory for the clinical use of the
IELISA; however, the coefficient might be improved by quantification of metabolites, such as M3 or other less common derivatives.

In summary, this immunoassay for diamidine compounds demonstrates utility in compound screening, pre-clinical testing, and as a clinical test. The assay uses a convenient format, tolerates medium and plasma as a sample matrix and has more than sufficient sensitivity to detect clinically relevant (and certainly toxic) levels of compound in blood. The IELISA is simpler and more cost-effective then the common small molecule assays, HPLC and LC/MS/MS, making it more easily adaptable for use in therapeutic drug monitoring in developing countries and other areas without access to specialized equipment; locations where HAT is of greatest concern. Although the current format of this assay lends itself primarily to a laboratory research setting, the IELISA could be modified to a simple colorchange assay for monitoring compound concentrations at point-of-care facilities. A further application of the monoclonal antibody is the visualization of diamidine compounds in tissue using immunohistochemical techniques providing a convenient and rapid approach to localizing sites of compound accumulation (unpublished results).

\section{REFERENCES}

1. Chappuis F, Loutan L, Simarro P, Lejon V, Buscher P. Options for field diagnosis of human african trypanosomiasis. Clin Microbiol Rev 2005;18:133-146.

2. Kennedy PG. Diagnostic and neuropathogenesis issues in human African trypanosomiasis. Int J Parasitol 2006;36:505-512.

3. Barrett MP, Boykin DW, Brun R, Tidwell RR. Human African trypanosomiasis: pharmacological re-engagement with a neglected disease. Br J Pharmacol 2007;152:1155-1171.

4. Kennedy PG. The continuing problem of human African trypanosomiasis (sleeping sickness). Ann Neurol 2008;64:116-126.

5. Mdachi RE, Thuita JK, Kagira JM, et al. Efficacy of the novel diamidine compound 2,5-Bis(4-amidinophenyl)- furan-bis-OMethlylamidoxime (Pafuramidine, DB289) against Trypanosoma brucei rhodesiense infection in vervet monkeys after oral administration. Antimicrob Agents Chemother 2008;53:953-957.

6. Pholig G, Bernhard S, Blum J, et al. Phase 3 Trial of Pafuramidine Maleate (Db289), A Novel, Oral Drug, for Treatment of First Stage Sleeping Sickness: Safety and Efficacy. Annual Meeting of the American Society for Tropical Medicine and Hygiene. New Orleans, 2008.

7. Wenzler T, Boykin DW, Ismail MA, Hall JE, Tidwell RR, Brun R. New treatment option for second stage African sleeping sickness: in vitro and in vivo efficacy of aza analogs of DB289. Antimicrob Agents Chemother 20:20, 2009.

8. Reisner HM, Gray DR, Jones SK, Rose BG, Tidwell RR. Immunoassays for pentamidine and related compounds: development of a facile inhibitory ELISA suitable for clinical use. J Clin Lab Anal 2000;14:73-82.

9. Kearney JF, Radbruch A, Liesegang B, Rajewsky K. A new mouse myeloma cell line that has lost immunoglobulin expression 


\section{Goldsmith et al.}

but permits the construction of antibody-secreting hybrid cell lines. J Immunol 1979;123:1548-1550.

10. Koren HS, Handwerger BS, Wunderlich JR. Identification of macrophage-like characteristics in a cultured murine tumor line. J Immunol 1975;114:894-897.

11. Nordan RP, Potter M. A macrophage-derived factor required by plasmacytomas for survival and proliferation in vitro. Science 1986;233:566-569.

12. Liu X, LeCluyse EL, Brouwer KR, et al. Biliary excretion in primary rat hepatocytes cultured in a collagen-sandwich configuration. Am J Physiol 1999;277:G12-G21.

13. Midgley I, Fitzpatrick K, Taylor LM, et al. Pharmacokinetics and metabolism of the prodrug DB289 (2,5-bis[4-(N-methoxyamidino)phenyl]furan monomaleate) in rat and monkey and its conversion to the antiprotozoal/antifungal drug DB75 (2,5-bis(4-guanylphenyl)furan dihydrochloride). Drug Metab Dispos 2007;35:955-967.

14. Trendler KL, Allen JL, Hall JE, et al. Quantification of the prodrug DB289 and an active metabolite, DB75, in rat and monkey plasma using SPE, liquid chromatography and electrospray ionization tandem mass spectrometry. Abstract 2212, 2000.

15. Patrick DA, Hall JE, Bender BC, et al. Synthesis and anti-Pneumocystis carinii pneumonia activity of novel dicationic dibenzothiophenes and orally active prodrugs. Eur J Med Chem 1999;34:575-583.

16. Generaux C, Bridges AS, Ismail MA, et al. Activation of the Antiparasitic Drug DB829: Structural Elucidation of Metabolites and Biotransformation Pathway in Human and Rat Liver Microsomes. Annual Meeting of the Globalization of Pharmaceutics Education Network. Belgium: Leuven, 2008. 